\title{
Baclofen Toxicity Causing Acute, Reversible Dyskinesia
}

\author{
Matthew T. Niehaus ${ }^{1}$ - Nicole C. Elliott ${ }^{1}$ - Kenneth D. Katz ${ }^{1,2}$
}

Received: 29 March 2016 /Revised: 5 May 2016/Accepted: 9 May 2016/Published online: 27 May 2016

(C) American College of Medical Toxicology 2016

\begin{abstract}
The following unique case demonstrates an episode of acute dyskinesia secondary to oral baclofen toxicity. We discuss an 80-year-old man with a history of Stage III chronic kidney disease, coronary artery disease, diabetes and stroke who presented to the Emergency Department with new onset of behavioral changes and irregular jerking movements. The patient had been recently prescribed baclofen $10 \mathrm{mg}$ twice daily for a back strain he suffered; he subsequently was admitted to the hospital, and his symptoms resolved within 48 hours of admission and discontinuance of baclofen.
\end{abstract}

Keywords Oral baclofen toxicity · Acute dyskinesia . Neurologic adverse effects $\cdot$ Reduced GFR

\section{Question}

An 80-year-old man with a medical history of stage III chronic kidney disease, coronary artery disease, diabetes, and stroke was prescribed a new medication for a low back sprain and developed the condition shown in the following video. What is the etiology of the patient's clinical presentation?

Kenneth D. Katz

Kenneth_D.Katz@1vhn.org

1 Department of Emergency Medicine, Lehigh Valley Health Network, Cedar Crest Boulevard \& I-78, Allentown, PA 18103, USA

2 Lehigh Valley Health Network, EM Research, 4th Floor, South Wing, 2545 Schoenersville Road, Bethlehem, PA 18017, USA

\section{Clinical Narrative}

An 80-year-old man with history of stage III chronic kidney disease, coronary artery disease, diabetes, and stroke presented to the emergency department with new onset of behavioral changes and irregular jerking movements. He had been recently prescribed baclofen $10 \mathrm{mg}$ twice daily for a back strain suffered 1 week prior and was taking it as prescribed. Other prescribed medications included aspirin $81 \mathrm{mg}$ daily, vitamin D3 2000 units daily, clopidogrel bisulfate $75 \mathrm{mg}$ daily, insulin aspart, 8 units with breakfast, 14 with lunch, and 12 with dinner, insulin glargine 35 units daily, lisinopril $2.5 \mathrm{mg}$ daily, loratadine $10 \mathrm{mg}$ daily, magnesium $84 \mathrm{mg}$ daily, metoprolol $12.5 \mathrm{mg}$ twice daily, pravastatin sodium $20 \mathrm{mg}$ daily, prednisone $7.5 \mathrm{mg}$ daily, and sitagliptin phosphate $50 \mathrm{mg}$ daily.

Physical examination revealed continuous, involuntary flexion, and extension movements of the upper and lower extremities, as well as repeated, involuntary turning of the head. A serum baclofen concentration measured upon presentation was $0.16 \mathrm{mcg} / \mathrm{mL}(0.08-0.40 \mathrm{mcg} / \mathrm{mL})$. The patient was admitted to the hospital, and his symptoms resolved within $48 \mathrm{~h}$ of admission and discontinuance of baclofen. None of his routinely prescribed medications was changed or found to have known interactions with baclofen, and no other acute medical condition was revealed.

This unique case demonstrates an episode of acute dyskinesia secondary to oral baclofen toxicity. Baclofen is a gamma-amino butyric acid (GABA) derivative that acts as an agonist at the $\mathrm{GABA}_{\mathrm{B}}$ receptor inducing presynaptic motor neuron inhibition and a central antispastic response [1]. While baclofen has been used primarily to limit spasticity in spinal cord disorders, it also has been studied as an inhibitor of dopamine reward pathways to treat drug abuse [2]. Baclofen is lipophilic, readily crosses the blood-brain barrier, has an elimination half-life of approximately 2 to 6 hours, and primarily is 
cleared via renal excretion [1]. Clearance, therefore, will be decreased in patients with a reduced glomerular filtration rate (GFR). Elderly patients may have seemingly normal creatinine, but with a marked reduction in GFR due to an overall lack of muscle mass and creatinine generation. Moreover, this patient had known chronic kidney disease with a baseline creatinine between 1.4 and $1.6 \mathrm{mg} / \mathrm{dL}$ and a GFR of 39 $47 \mathrm{~mL} / \mathrm{min}$, placing him at increased risk for decreased drug clearance even at therapeutic levels of the drug.

This patient developed altered mental status and dyskinesia in the setting of therapeutic oral baclofen initiation, which is exceedingly rare, documented in only one other case report. Ryan, et al., reported the onset of dyskinesia in a 75-year-old man approximately 4 days after baclofen treatment was initiated. The patient in that case report had a documented creatinine of $1.4 \mathrm{mg} / \mathrm{dL}$; however, no reference range or GFR was reported. Baclofen was implicated as the causative agent since the patient's symptoms resolved after discontinuation of the drug and then reappeared with the re-institution of oral baclofen therapy (15 $\mathrm{mg}$ daily) [3].

The exact mechanism of baclofen-induced dyskinesia is poorly understood. Classically, drug-induced dyskinesia has been reported with the use of dopamine blocking agents (DBA). It has been postulated that the relative dopaminergic hypofunction produced by DBAs causes overactivity of cholinergic mechanisms [3]. With respect to GABA receptors, rodent models have shown that $\mathrm{GABA}_{\mathrm{B}}$ receptors are expressed on both dopamine neurons, as well as GABA neurons [2]. In rats exposed to baclofen, it was shown that activation of $\mathrm{GABA}_{\mathrm{B}}$ receptors inhibited both GABAergic and dopaminergic neurons, and decreased dopamine release [2]. Furthermore, there have been reports of baclofen neurotoxicity in patients with pre-existing cerebral lesions, which may affect receptor activity and function [1]. Interestingly, our particular patient's prescribed medications included zolpidem, which when used in conjunction with baclofen may increase sedation. It has been suggested in the literature that concomitant use of central nervous system depressants may affect the concentration at which baclofen may become toxic in patients with renal disease [1]. Baclofen was not found to have any other overt drug-drug interactions.

\section{Compliance with Ethical Standards}

Informed Consent Consent for publication of this case was obtained and provided to the journal in accordance with JMT policy.

Conflicts of Interest The authors declare that they have no conflicts of interest.

Sources of Funding The authors have no outside support information.

\section{References}

1. El-Husseini A, Sabucedo A, Lamarche J, Courville C, Peguero A. Baclofen toxicity in patients with advanced nephropathy: proposal for new labeling. Am J Nephrol. 2011;34(6):491-5.

2. Cruz HG, Ivanova T, Lunn ML, Stoffel M, Slesinger PA, Lüscher C. Bi-directional effects of GABAB receptor agonists on the mesolimbic dopamine system. Nat Neurosci. 2004;7(2):153-9.

3. Ryan DM, Blumenthal FS. Baclofen-induced dyskinesia. Arch Phys Med Rehabil. 1993;74(7):766-7. 